\title{
La simplificación neopositivista del lenguaje de la psicopatología desde una perspectiva post-wittgensteiniana.
}

Postwittgensteinian perspective on the neopositivistic oversimplification of psychopathological language.

\author{
Ferrán Molins Gálvez a , José Manuel López-Santín b \\ ${ }^{a}$ Psiquiatra. Centres Assistencials Emili Mira i Lopez. Parc de Salut Mar. Santa Coloma de Gramanet, Bar- \\ celona, España. ${ }^{b}$ Psiquiatra. Centre de Salut Mental Martí i Julià. Parc de Salut Mar. Barcelona, España.
}

Correspondencia: Ferran Molins Galvez, (ferran.galway@gmail.com)

Recibido: 10/03/2014; aceptado con modificaciones: 24/06/2014

\begin{abstract}
RESUMEN: La deriva neopositivista y operacional en psiquiatría se ha acompañado de un recorte de la terminología psicopatológica. El criterio de selección seguido, consistiría en conservar solamente aquellos términos que se refieran a fenómenos mentales cuyas descripciones puedan ser reducidas al menor nivel posible de inferencia, a descripciones del comportamiento externo o incluso a un lenguaje ordinario. En relación a este criterio, se discuten cuatro cuestiones desde una perspectiva post-wittgensteiniana: la posibilidad de un lenguaje observacional puro, la ausencia de un marco teórico único en la psicopatología, la presencia de metáforas y analogías en el lenguaje de la psicopatología y la discusión acerca de si las distinciones que establece la psicopatología representan entidades naturales.
\end{abstract}

PALABRAS CLAVE: Psiquiatría, Psicopatología, Filosofía de la ciencia, Filosofía del lenguaje, Wittgenstein.
ABSTRACT: Neopositivism and operationalism in psychiatry has promoted a simplification of the psychopathological language. According to its selection criteria, the psychopathological terms that should be kept are those whose descriptions can be done in the lowest possible level of inference, in descriptions of external behavior, or else in simple lay language. Four issues are discussed in relation to this criteria from a post-wittgensteinian perspective: the possibility of a pure observational language, the absence of a unique theoretical framework in psychopathology, the presence of methapors and analogies as well as the question about the representational nature of the psychopathological language.

KEY WORDS: Psychiatry, Psychopathology, Philosophy of science, Philosophy of language, Wittgenstein.

Agradecimientos:

Al profesor Román Cuartango, de la facultad de filosofia de la Universitat de Barcelona, por sus comentarios y supervisión.

\section{Introducción}

Los textos que tratan la historia de la psiquiatría suelen describir el momento en que aparecieron determinados términos psicopatológicos así como su destino, ya fuera cambiar de sentido, mantenerse o caer en desuso hasta perderse en el olvido. La negligencia de los aspectos históricos de una disciplina como la psiquiatría puede generar la idea de que el lenguaje tal y como lo encontramos actualmente en los manuales al uso es fruto de un progresivo refinamiento y mejor adecuación a la realidad, 
como si progresivamente se hubiera obtenido una mejor fotografía de la realidad. Lo que suele quedar en la sombra son los criterios, las condiciones y las razones que propician la generación, el mantenimiento o el abandono de determinados términos.

Este trabajo se propone aportar argumentos contra el criterio de selección, plasmado en el manual DSM, que se ha promovido desde que las aproximaciones neopositivistas, operacionales y eliminativistas parecen haber tomado las riendas de la disciplina. Según este criterio solamente sería adecuado conservar aquellos términos psicopatológicos que se refieran a fenómenos mentales cuyas descripciones puedan ser reducidas al "menor nivel posible de inferencia", idealmente a descripciones del comportamiento externo o incluso a un lenguaje ordinario (el DSM lo puede leer un no especialista) (1). Se esperaría así, que los clínicos usaran consistentemente lenguaje ordinario de primer orden, para enmarcar definiciones imparciales, acontextuales y ateóricas que mejoraran la fiabilidad de las descripciones. El objetivo perseguido sería aumentar la fiabilidad entre observadores y establecer un mínimo lenguaje común entre escuelas diversas. Aunque ambas pretensiones tienen aspectos positivos, seguir tal criterio de selección encuentra problemas importantes.

La cuestión que se plantea es: ¿está justificada la reducción y simplificación del lenguaje de la psicopatología a un lenguaje cuyos términos puedan reducirse meramente a lo observacional? Es decir, ¿es legítima la simplificación y eliminación de conceptos del lenguaje psicopatológico que las aproximaciones neopositivistas y operacionales parecen promover?

Cuatro son los argumentos que se discuten al respecto: la posibilidad de un lenguaje observacional puro, la ausencia de un marco teórico único en la psicopatología, la presencia difícilmente evitable de metáforas y analogías en el lenguaje de la psicopatología y la discusión acerca de la necesidad de una ontología de los trastornos mentales, es decir, de que las distinciones que establece la psicopatología, reflejen entidades naturales.

\section{2-La posibilidad de un lenguaje observacional puro}

Los filósofos de la ciencia, durante el siglo XX, se han visto obligados a revisar los principios del empirismo clásico(2). Uno de los supuestos empiristas que ha sido revisado en el desarrollo de la filosofía de la ciencia en el siglo XX, es el que afirma que la base observacional, es la que proporciona la experiencia "neutra" con la cual contrastar las afirmaciones de la teoría. Se pensaba que las teorías contenían dos tipos de términos, los términos observacionales y los términos teóricos. El principal motivo de la introducción de la distinción teórico/observacional era proporcionar legitimidad semántica, según los criterios empiristas, a los términos "sin conexión 
empírica inmediata" que las teorías científicas introducen a través de sus leyes para dar cuenta de los fenómenos. Esta finalidad semántica iba acompañada de otra metodológica, pues se pretendía que la base observacional es la que proporciona la experiencia "neutra" con la cual contrastar las afirmaciones de la teoría. Esta neutralidad teórica de la base de contrastación parece en primera instancia fundamental, pues de lo contrario parecería que la teoría resulta autojustificativa. Si la experiencia observacional que se usa para contrastar la validez de una teoría fuese dependiente de la teoría en cuestión, esto es, si la elaboración de informes observacionales que sirven de base de contrastación presupusiera la validez de la teoría, entonces tendríamos un círculo autojustificativo. Por tanto, la base observacional, si ha de servir para la contrastación, debe ser teóricamente neutral. La distinción entre términos teóricos y términos observacionales parece asimismo problemática en la medida que lo que consideramos usualmente como observaciones requieran adiestramiento o algún tipo de conceptualización teórica.

Diversos autores han rechazado la idea que la observación esté libre de conceptualización teórica. Duhem (3) constató que todo informe observacional, supone una interpretación de los datos de los sentidos, y una interpretación no es más que una conceptualización teórica, sea explícita o implícita. Aun así, reconocía que el aparato conceptual interpretador que genera la base observacional no corresponde a cierta teoría que usa dicha base en la contrastación, pero corresponderá a otro "constructo teórico"; este constructo presupondrá a su vez otro en la descripción de sus propios fenómenos empíricos y así sucesivamente.

El rechazo del privilegio que el empirismo tradicionalmente había dado a los enunciados observacionales, mostrando que dependen de algún tipo de conceptualización o teoría previa es uno de los aspectos del debate filosófico contemporáneo entre fundamentistas y coherentistas (4). Las posiciones fundamentistas parten del reconocimiento de algún tipo de representación o creencia privilegiada que sea autojustificadora. El empirismo, como posición fundamentista, anhelaba fundamentar y justificar todo nuestro conocimiento en la experiencia sensorial, en los enunciados observacionales, en las creencias perceptivas. Todo conocimiento (empírico) empezaba con las afecciones de nuestro entorno sobre nuestro aparato sensorial y toda justificación del mismo, debía apelar en última instancia a esa "observación directa" del entorno. Pero este supuesto no pudo ser mantenido por mucho tiempo. Y por otro lado, de este supuesto razonable, no se sigue que la justificación de cada pieza de nuestro conocimiento deba proceder del mismo modo, es decir, apelando a la observación.

Las posiciones coherentistas, en cambio, han optado por suavizar la distinción entre enunciados teóricos y enunciados observacionales. Sostienen que no hay enunciados privilegiados, irrebatibles o no revisables, e insisten en que la justificación de una creencia (p.ej. la creencia de que "El paciente que tengo enfrente está ansioso") 
no puede predicarse de una creencia aislada sino que es, más bien, una propiedad poseída por un sistema global de creencias. En el caso de las creencias perceptivas, las creencias sobre enunciados observacionales, la justificación no depende exclusivamente de lo que acontece a nuestro alrededor y del funcionamiento correcto de nuestros órganos sensoriales, sino de una red de habilitaciones y compromisos(5). Mi creencia de que "El paciente que tengo enfrente está ansioso" presupone otras creencias y habilitaciones mías, tales como, que las condiciones de mi percepción no son anormales, que soy capaz de distinguir la ansiedad de otros estados de ánimo, etc. La justificación de estas creencias respecto a mis competencias como agente epistémico remite a su vez a nuevas creencias, y así se va tejiendo una compleja red de interrelaciones de apoyo.

\section{3-Ausencia de un marco teórico único}

En segundo lugar y ligado con el anterior, cabe señalar que uno de los rasgos que caracteriza la psicopatología es la ausencia de un marco teórico único para definir los conceptos(6). La presencia de múltiples escuelas psicológicas y contextos asistenciales diversos favorece que un mismo término psicopatológico adopte sentidos distintos. La extensión de un término psicopatológico según una teoría o modelo psicopatológico puede así, coincidir nada o sólo parcialmente con la extensión del mismo término según otro modelo. El número de distinciones y categorías que las diversas psicopatologías establecen en relación a la "locura", así como el significado de éstas, no se fundamenta en la identificación de géneros naturales claramente delimitados y corroborados por pruebas de elevada validez y fiabilidad. Estas distinciones se sustentan en modelos teóricos con orígenes histórico-sociales diversos generados probablemente en contextos asistenciales diversos. Las consecuencias de obviar la dependencia del marco teórico es que, fenómenos clínicos heterogéneos acaben confluyendo en un mismo término.

Pero la solución a esta pluralidad de marcos teóricos no parece ser el recorte del lenguaje de la psicopatología o tratar de imponer uno por encima de los demás. En cualquier caso, y dadas las características complejas de las cuestiones con que se tratan parece prudente mantenerse abierto a las aportaciones de los diversos marcos teóricos aceptando el valor de la pluralidad. Tampoco parece adecuado concluir que existe una inconmensurabilidad absoluta entre modelos teóricos y posiblemente, los puntos de acuerdo son más de los que habitualmente se quieren aceptar. Por otro lado, dada la importancia del poder que la sociedad delega en la psiquiatría y los dispositivos de salud mental, esto es, la de establecer límites y distinciones en el pantanoso ámbito de la "normalidad" y "funcionalidad" psicológica, parece aconsejable que los 
ORIGINALES Y REVISIONES

discursos teóricos que se generan, estén abiertos al diálogo público incluyendo en la medida de lo posible, los propios afectados y sus familias.

\section{4-La presencia de metáforas y analogías}

El examen del lenguaje psicopatológico también muestra algunas de las estrategias que la psicopatología ha desarrollado para expresar y dar cuenta de fenómenos diversos, entre los que se han destacado el uso de las metáforas y las analogías. Esta característica, desde ciertas posiciones cientifistas, se ha considerado problemática(7). Los diversos marcos teóricos de la psicopatología han explotado las posibilidades de determinadas metáforas para dar cuenta de diversos fenómenos de la vida psíquica.

"El paradigma psicoanalítico usaba inicialmente como hilo conductor un modelo termodinámico o hidrodinámico, basado en la comparación metafórica de la psique con una máquina de vapor, lo que explica el uso de nociones como la de represión (la presión del gas que sale por las junturas). Freud sabía ya que el cerebro se compone de neuronas (Cajal acababa de descubrirlo) y que éstas transmiten electricidad. Recogió de la física la idea de energía como cantidad conservada y la adoptó como "energía psíquica"(7).

"Además, el desarrollo imparable de la tecnología de la computación y de los computadores empezó a ejercer una creciente fascinación y a sugerir un nuevo paradigma. La cibernética y la inteligencia artificial fueron los dos primeros desarrollos en esta dirección"(7).

Una historia completa de la psicología y la psicopatología debiera recoger la influencia que el lenguaje tecnológico ha tenido en la elaboración y generación de modelos teóricos psicológicos y psicopatológicos. La mente ha sido pensada a partir de dispositivos tales como el telégrafo, la radio, la cámara de cine, y más recientemente el computador. Los modelos cibernéticos de matemáticos como Norbert Wiener ${ }^{1}(8-9)$, al mismo tiempo que han llevado al desarrollo de las redes de sistemas computacionales globales como Internet, han inspirado modelos tan dispares como la escuela de psicología sistémica de Palo Alto, o las más recientes neurociencias cognitivas. El hombre anhela tanto construir una imagen de sí mis$\mathrm{mo}^{2}(10)$, como una imagen inteligible y manejable de aquello que supuestamente lo gobierna, ya sea el alma, la mente o el cerebro.

\footnotetext{
Norbert Wiener (1894-1964) fue un matemático estadounidense fundador de la cibermética. En dos de sus obras propone extrapolar algunas de sus teorías al estudio de la psicopatología.

Peter Sloterdijk (1947- ) plantea la presencia de metáforas tecnológicas ya en el relato bíblico del Génesis. Según esta interpretación, el ser humano es presentado como el producto de un maestro ceramista (Dios) capaz de insuflar vida, y la historia estaría marcada por la distancia que separa la humano-técnica de la teo-técnica. Véase: Sloterdijk P. Esferas. 1a ed. Vol.1, Burbujas: microesferología. Madrid: Siruela; 2003. 583 p.
} 
Los mitos y la tragedia clásica han sido otra fuente de analogías y metáforas, en algunos marcos teóricos psicopatológicos. Para James Hillman (11) la metapsicología psicoanalítica puede leerse como un relato mítico que describe la historia de las tensiones y los conflictos entre las clásicas entidades intrapsíquicas postuladas: el "ello", el "yo" y el "superyo".

Inversamente, algunos autores (12), han reprochado recientemente a la neurociencia cognitiva contemporánea una falaz apropiación de conceptos psicológicos tales como: "creer", "querer", "percibir". Según estos autores, la neurociencia cognitiva habría caído, más o menos inadvertidamente, en la denominada falacia mereológica que consiste en atribuir a las partes propiedades que sólo son atribuibles al todo. Es decir, mediante un antropomorfismo ilegítimo según estos autores, se habrían transferido facultades personales a entidades subpersonales tales como las neuronas, los circuitos y redes neuronales o el cerebro entero.

Por otro lado, el examen de los términos de la psicopatología muestra que muchos de ellos conservan los ecos de un origen metafórico. Recorrer los glosarios de la psicopatología se asemeja a pasearse por un cementerio de metáforas marchitas, ateniéndose a la imagen nietzscheana del lenguaje. Muchas de estas expresiones metafóricas están ya presentes en los lenguajes naturales (13-14), pero la psicopatología ha explotado sus posibilidades inferenciales. Metáforas más o menos acertadas que dejan entrever las múltiples y diversas tentativas de engendrar conceptos y expresiones para captar la experiencia ajena y hacerla transmisible. La conciencia, el yo, la afectividad, el pensamiento y el lenguaje, son aspectos del lenguaje psicopatológico ricos en metáforas. En relación a la conciencia, encontramos términos tales como: "el nivel de conciencia", "los estados crepusculares", "la obnubilación de la conciencia" que sacan provecho de las imágenes que evocan las metáforas que toman la conciencia como un fluido o como una fuente de luz. En cuanto al "yo", encontramos términos que lo presenta como una entidad "física" con determinadas propiedades: "actividad del yo", "identidad del yo", "vitalidad del yo", "fragilidad del yo", "rigidez del yo", "debilidad del yo", "demarcación del yo", "consistencia del yo". La afectividad también se presenta bien como una entidad sólida con propiedades físicas (p.ej. "ansiedad flotante", "afecto aplanado", "labilidad afectiva"), como un sonido (p.ej. "armonía o sintonía emocional") o como un fluido (p.ej. "incontinencia emocional"). Finalmente, en el ámbito del pensamiento y el lenguaje se usan términos que participan de metáforas que los hacen aparecer como una entidad objetiva (p.ej. "robo e inserción del pensamiento", "pensamiento circunferencial", "pensamiento tangencial", "pérdida de objetivo del pensamiento", "torpeza cognitiva") a una onda electromagnética (p.ej. "retransmisión del pensamiento") o a un fluido (p.ej. "fuga de ideas", "presión del pensamiento", "fusión y condensación del pensamiento"). El lenguaje de la psicopatología, visto desde esta perspectiva, contiene términos que posiblemente surgieron de una metáfora acertada. El objeto de la experiencia psico- 
patológica, la experiencia ajena, es un material friable, que promueve los esfuerzos para generar conceptos que permitan orientarse.

La analogía y la metáfora, lejos de ser meros recursos para embellecer y ornamentar un discurso o para facilitar el aprendizaje de determinados aspectos abstractos de una materia de conocimiento, incitan formas creativas de inteligibilidad, redescripciones de nuestra experiencia que nos sean más provechosas, especulaciones teóricas atrevidas y la generación de nuevas hipótesis. Los paradigmas metafóricos en la psicología y la psicopatología han sido y siguen siendo útiles en el dominio de la experiencia y práctica clínica psiquiátrica. La sustitución prematura de los paradigmas metafóricos en psiquiatría, por un supuesto lenguaje literal y representacional (si es que tal concepción del lenguaje es todavía sostenible), puede contraer el riesgo de empobrecer y encorsetar la experiencia psiquiátrica así como deformar su objeto.

El desarrollo de teorías y modelos basados en analogías y metáforas ha sido una constante en la especulación de la ciencia y especialmente en la psicología y la psicopatología. Los modelos metafóricos, frecuentemente, no son fácilmente reducibles a un lenguaje "materialista", pero no por ello, se debería concluir que son meras ficciones desechables. En todo caso, en muchos casos deben ser consideradas ficciones útiles, con valor heurístico, para orientar la práctica y la investigación. La fidelidad a una perspectiva materialista sobre lo que existe en el mundo, no debiera encorsetar de buenas a primeras, los esfuerzos de producir modelos para adquirir un mayor inteligibilidad en la experiencia psicopatológica.

\section{5-El estatuto ontológico de los conceptos de la psicopatología}

La cuarta de las cuestiones vinculadas con la simplificación del lenguaje psicopatológico concierne al estatuto de sus conceptos psicopatológicos y lo que la ontología de lo mental tiene que decir al respecto. El espíritu de los tiempos que corren, aboga por un eliminativismo materialista, es decir, por la reducción del lenguaje de la psicología al lenguaje de la neurofisiología y la neurobiología.

La discusión de esta cuestión requiere un preámbulo. Durante el siglo XX, tanto en la tradición de la filosofía analítica como en la tradición continental, se asiste a un ataque a la idealidad del sentido:

"La reacción antihusserliana continental y la reacción antifregeana analítica coinciden en una intencionalidad común, la de asestar un golpe a la idealidad inmaculada del sentido (o del significado), y por así decirlo, 'mundanizarla"(15). 
Wittgenstein, en las Investigaciones Filosóficas(16), mostró las paradojas del esquema representacional del significado (también denominado teoría ideacional del significado(17)), según el cual, la inteligibilidad de la realidad se garantiza porque los signos/palabras representan, es decir, están por los objetos de una realidad independiente por mediación de representaciones/conceptos/ideas (éstas ideas se encontrarían o bien en las mentes de los sujetos al modo de los empiristas ingleses como Locke o bien en un reino de entidades ideales como en Platón). Wittgenstein mostró, que la denotación, es un juego lingüístico más, dentro de los múltiples y diversos juegos de lenguaje posibles(18). La noción de sentido, de significado de un signo, se abría entonces a otros posibles juegos de lenguaje, y se terminaba con el monopolio que había detentado el juego de la denotación en relación a la noción de sentido en gran parte de la tradición filosófica empirista. Como Manfred Frank señala:

"[Para el Wittgenstein de las Investigaciones Filosóficas], comprender el significado de una palabra o una cadena de ellas (un enunciado) no significa saber qué cosas o estados de cosas son representados (o designados) por las palabras. Porque una parte considerable de las acciones lingüísticas no consiste en hacer enunciados sobre el estado de cosas existente en una porción de la realidad. Existe, por ejemplo, el dar órdenes, el postular hipótesis, el inventar historias, el hacer teatro, el cantar rondas, el adivinar enigmas, el contar chistes, el traducir un idioma a otro, el pedir, el agradecer, maldecir, saludar, rezar, etc.; en pocas palabras, formas del acto de habla en las cuales no se hace una referencia de constatación hacia la realidad. De esto, Wittgenstein deduce que nosotros entendemos las palabras o los enunciados que se utilizan en los 'juegos lingüísticos no constatativos, y cuyo significado no puede consistir en la adecuación a un hecho (transemiológico), sino en la forma de su utilización, en la medida en que esta utilización, en su regularidad, ayuda a expresarse a una 'forma de vida' colectiva. El significado de una palabra/un enunciado es por tanto la forma de su uso en contextos de acción convencionalizados, es decir, socialmente determinados: hablar es una forma de acción social, a saber, la de comunicar; su regularidad remite a la praxis social que la subyace. Como formas de vida, las acciones son ipso facto intersubjetivas, en el sentido de que están codificadas. Solamente en ello se fundamenta su posibilidad transindividual de ser comprendidas" (19).

El lenguaje de la denominada psicología popular está formado por múltiples juegos de lenguaje que no tienen por qué tener un equivalente unívoco en el lenguaje de la neurobiología. Según Wittgenstein uno de los errores que se comete en las discusiones sobre la ontología de lo mental y la relación mente-cuerpo, es aceptar la idea de que cuando alguien expresa algo de sí mismo como por ejemplo "Tengo dolor", está buscando describir un estado mental. Para Wittgenstein dichas expresiones deben entenderse dentro de determinados juegos lingüísticos como gestos para con- 
seguir efectos prácticos en el entorno. El punto crucial es, cómo los demás reaccionan a tales expresiones y qué uso se hace de las mismas llevando a cabo determinadas acciones y respetando determinadas costumbres. La lección que se puede obtener de los últimos trabajos sobre filosofía de la psicología de Wittgenstein es que nuestras expresiones psicológicas no son usadas primeramente para hacer enunciados descriptivos de nuestros estados y procesos mentales. Cuando uno habla de tener dolor o de estar asustado no trata de describirlo, trata de ser atendido o de ser consolado. Incluso si alguien consiguiera sustituir todo el vocabulario psicológico por un conjunto de términos manejables relativos a entidades corporales, esto no nos diría nada especial sobre nuestros conceptos psicológicos, porque lo que significan se muestra en nuestros juegos de lenguaje, esto es, en nuestras acciones.

La misma cuestión es aplicable a los términos de la psicopatología. El sentido de las distinciones y los términos psicopatológicos lo adquieren en relación a los juegos lingüísticos que se ponen en marcha en los diversos contextos de la práctica psiquiátrica. Cuando por ejemplo, un profesional es informado de que ha acudido a urgencias una persona con un diagnóstico de trastorno antisocial de la personalidad, el principal interés de la etiqueta para el profesional es práctico. El concepto diagnóstico le ayuda a orientar el trato que debe tener ante el caso, tomar las precauciones debidas, las actitudes, el lenguaje y el tono de voz adecuado para poder mantener una actitud de ayuda mientras sale airoso del encuentro. La correspondencia de tal etiqueta diagnóstica con algún tipo de referente natural es, en ese contexto, algo secundario.

En este sentido la validez de las distinciones psicopatológicas se justifica por la propia práctica clínica y si se diera el caso de que determinadas distinciones no corresponden con hallazgos neurobiológicos específicos, no por ello dejarían de ser útiles en determinados contextos de la práctica clínica. Dicho de otro modo, la legitimidad de las distinciones y términos psicopatológicos se justifica en gran medida por la propia práctica asistencial. Los hallazgos neurobiológicos que puedan descubrirse pueden ser de ayuda en determinados contextos de la práctica, pero insuficientes en otros. En cualquier caso, cabe insistir que el valor de determinados términos psicopatológicos, no reside en tratar de señalar hacia algún tipo de entidad natural, sino más bien, en el intento de capturar un fragmento de experiencia y hacerla comunicable entre una comunidad de profesionales.

Así pues, la operación de tratar de reducir el lenguaje de la psicología y la psicopatología al lenguaje de la neurofisiología parece más bien fruto de una concepción del lenguaje bajo un esquema puramente representacionista. Si se acepta que con lo que se trata es con distintos juegos de lenguaje, no parece que haya nada que reducir (20). 


\section{6-Conclusiones}

En primer lugar, se ha tratado de aportar argumentos para mostrar que el criterio de selección neopositivista de los términos de la psicopatología no es adecuado dada la fuerte distinción que establece entre términos observacionales y términos teóricos. Se ha hecho patente así la relevancia de determinadas cuestiones filosóficas de fondo que conciernen al modo de entender el lenguaje de la psicopatología. La preocupación por obtener un lenguaje descriptivo como fundamento de una práctica psiquiátrica científica y lengua común de todos los profesionales de la salud mental ha sido una preocupación constante desde pioneros de la psiquiatría como Phillipe Chaslin o Karl Jaspers. El positivismo lógico se preocupó intensamente de establecer los requisitos que debía cumplir un lenguaje para considerarse científico, asumiendo supuestos empiristas. El modo de concebir el lenguaje y su relación con el mundo desde la perspectiva positivista, es lo que la filosofía contemporánea ha denominado esquema representacionista, según el cual, el lenguaje con sentido es el que se puede referir a hechos del mundo, que en un extremo se fundamentan en las impresiones sensoriales del sujeto. Este esquema como se ha tratado de mostrar tuvo que ser revisado. La filosofía contemporánea, tras el giro lingüístico y pragmatista, ha tratado de superar el esquema representacionista del lenguaje, abriendo la posibilidad de establecer condiciones de legitimidad para los discursos que no cumplen con las restrictivas condiciones de objetividad que impone la perspectiva positivista tradicional.

Las dificultades para separar los hilos que entrelazan observación y teoría, experiencia y conceptualización, lleva a aporías irresolubles las pretensiones de los manuales diagnósticos modernos de ofrecer descripciones ateóricas. La dependencia que las definiciones de los términos de la psicopatología mantiene con los marcos teóricos parece difícilmente evitable y en cualquier caso, mientras no se adopten actitudes dogmáticas respecto un único marco teórico, y se esté dispuesto a revisar o desechar teorías, no debiera ser visto como un obstáculo para el desarrollo científico.

Asimismo la distinción entre lenguaje literal y lenguaje metafórico parece difuminarse ante el examen de muchos términos psicopatológicos. Lejos de ver las metáforas como algo de lo que todo lenguaje científico debería desprenderse, cabe preguntarse si no forman parte del mismo motor que promueve redescripciones productivas de la realidad.

Finalmente se ha puesto de manifiesto que las condiciones de legitimidad de las distinciones del lenguaje de la psicopatología se dan en gran parte en el contexto asistencial, es decir, en la práctica clínica, lejos de los laboratorios de investigación donde se abstraen muchos aspectos de la experiencia psiquiátrica. Y con esto, en tiempos donde los profesionales que se dedican exclusiva o la mayor parte de su tiempo a la actividad asistencial parecen estar infravalorados ante la figura de aquellos que se dedican mayormente a la gestión o a la investigación, reclama una reapropiación por parte de estos profesionales del apelativo 'experto'. 


\section{BIBLIOGRAFÍA}

(1) Parnas J, Sass LA, Zahavi D. Rediscovering psychopathology: the epistemology and phenomenology of the psychiatric object. Schizophr Bull. 2013; 39:270-7.

(2) Díez JA, Moulines CU. Fundamentos de filosofía de la ciencia. 3a ed. rev. Barcelona: Ariel; $2008.527 \mathrm{p}$.

(3) Duhem PM. La teoría física, su objeto y estructura. 1a ed. Barcelona: Herder; 2003. 444 p.

(4) Vega Reñón L, Olmos Gómez P, editors. Compendio de lógica, argumentación y retórica. 2a ed. rev. Madrid: Trotta; 2012. 712 p.

(5) Brandom R. La articulación de las razones: una introducción al inferencialismo. 1a ed. Madrid: Siglo XXI España; 2002. 288 p.

(6) Gorostiza PR, Manes JA. Misunderstanding psychopathology as medical semiology: an epistemological enquiry. Psychopathology. 2011;44:205-15.

(7) Mosterín J. La insuficiencia de los paradigmas metafóricos en psicología. Rev. Asoc. Esp. Neuropsiq., 2003; 23(85):89-104.

(8) Wiener N. Cibernética o el control y comunicación en animales y máquinas. 1a ed. Barcelona: Tusquets; $1985.268 \mathrm{p}$.

(9) Wiener N. Cibernètica i societat. Barcelona: Edicions 62; 1965.216 p.

(10) Sloterdijk P. Esferas. 1a ed. Vol.1, Burbujas: microesferología. Madrid: Siruela; 2003. 583 p.

(11) Hillman J. Healing Fiction: On Freud, Jung, Adler. New York: Spring Publications; 1994.148 p.

(12) Bennett MR, Hacker PM. Philosophical foundations of neuroscience. Malden(MA): Blackwell; 2003. 461 p.

(13) Lakoff G, Johnson M. 1st ed. Philosophy in the flesh: the embodied mind and its challenge to Western thought. New York: Basic Books; 1999. 624 p.

(14) Kövecses Z. Metaphor and emotion: language, culture, and body in human feeling. 1st ed. Paris: Cambridge University Press \& Editions de la Maison des Sciences de l'homme; 2003. 223 p.

(15) Sáez Rueda L. Movimientos filosóficos actuales. 3a ed. Madrid: Trotta; 2009. 527 p.

(16) Wittgenstein L. Obras Wittgenstein I: Tractatus logico-philosophicus; Investigaciones filosóficas; Sobre la certeza. 1a ed. Madrid: Gredos; 2009. 830 p.

(17) García Suárez A. Modos de significar: una introducción temática a la filosofía del lenguaje. 2a ed. Madrid: Tecnos; 2011.726 p.

(18) Hadot P. Wittgenstein y los límites del lenguaje. 1a ed. Valencia: Pre-Textos; 2007. 144 p.

(19) Frank M. ¿Qué es el neoestructuralismo? 1a ed. México: Fondo de Cultura Económica; $2011.530 \mathrm{p}$.

(20) Schulte J. Experience and expression: Wittgenstein's philosophy of psychology. 1st ed. New York: Oxford University Press; 1995. 179 p. 